\title{
Combinations of Fuzzy Rough Covers and Covering Principles
}

\author{
D. Vidhya
}

\begin{abstract}
Rough set deals the mathematical approach to uncertain knowledge. It has many interesting applications in the area of machine learning, decision analysis, pattern recognition etc., A fuzzy rough set is a generalization of a rough set, derived from the approximation of a fuzzy set. The focus of fuzzy rough set is to define lower and upper approximation from the universal set. This paper gives four operations of open covers and three notions of covering principles in fuzzy rough set. Also investigates the relations of covers and principles. Finally combine the open covers and principles, find the relationship between them.
\end{abstract}

\section{Keywords-Fuzzy rough cover(s), covering principle(s)}

\section{INTRODUCTION}

Authors [1], [6] and [7] said the concepts and properties of fuzzy sets, applications of fuzzy set and fuzzy topological spaces. Further various uncertainities that arise in the real world problems are solved by using rough set theory $[3,5]$, intuitionistic fuzzy rough set theory [2] etc. S. Nanda and S. Majumdar [4] analysed the concept of fuzzy rough set. This paper introduces the concepts of types of fuzzy rough open cover and types of fuzzy rough covering principle. Some basic properties are also established. In particularly, the concept of relationship between them are discussed.

\section{PRELIMINARIES}

Definition 2.1 [4]

$F=\left(F_{L}, F_{U}\right)$ and $B=\left(B_{L}, B_{U}\right)$ in $Z$,

1) $\quad \mathrm{F}=\mathrm{B}$ if and only if $\mu_{\mathrm{F}_{\mathrm{L}}}(\mathrm{a})=\mu_{\mathrm{B}_{\mathrm{L}}}$ (a) for each $\mathrm{a} \in \mathrm{Z}_{\mathrm{L}}$ and $\mu_{\mathrm{F}_{U}}(\mathrm{a})=\mu_{\mathrm{B}_{U}}$ (a) for each $\mathrm{a} \in \mathrm{Z}_{\mathrm{U}}$.

2) $\quad \mathrm{F} \subseteq \mathrm{B}$ if and only if $\mu_{\mathrm{F}_{\mathrm{L}}}$ (e) $\leq \mu_{\mathrm{B}_{\mathrm{L}}}$ (e)for each e $\in \mathrm{Z}_{\mathrm{L}}$ and $\mu_{\mathrm{F}_{\mathrm{U}}}(\mathrm{e}) \leq \mu_{\mathrm{B}_{\mathrm{U}}}$ (e)for every e $\in \mathrm{Z}_{\mathrm{U}}$.

3) $\mathrm{C}=\mathrm{F} \cup \mathrm{B}$ if and only if $\left.\mu_{\mathrm{C}_{\mathrm{L}}}(\mathrm{r})=\max _{\left[\mu_{\mathrm{F}_{\mathrm{L}}}\right.}(\mathrm{r}), \mu_{\mathrm{B}_{\mathrm{L}}}(\mathrm{r})\right]$ for all $\mathrm{r} \in \mathrm{Z}_{\mathrm{L}}$ and $\mu_{\mathrm{C}_{\mathrm{L}}}(\mathrm{r})=\max \left[\mu_{\mathrm{F}_{\mathrm{L}}}(r), \mu_{\mathrm{B}_{U}}(\mathrm{r})\right]$ for all $r \in \mathrm{Z}_{\mathrm{U}}$.

$$
\begin{aligned}
& D=F \cap B \text { if and only if } \\
& \mu_{D_{L}}(f)=\min \left[\mu_{F_{L}}(f), \mu_{B_{L}}(f)\right] \text { for all } f \in Z_{L} \text { and } \\
& \mu_{D_{U}}(f)=\min \left[\mu_{F_{U}}(f), \mu_{U}(f)\right] \text { for all } f \in Z_{U} .
\end{aligned}
$$

A complete lattice $L$ is for any indexed set $J,\left\{H_{i}: i \in J\right\}$ is $F R S(Z), E=\cup_{i} H_{i}$ if and only if $\chi_{E_{L}}(r)=\sup _{i \in J} \chi_{H_{L_{i}}}(r)$ for all $r \in Z_{L}$ and $\chi_{E_{U}}(r)=\sup _{i \in J} \chi_{H_{U_{i}}}(r)$ for all $r \in Z_{U}$. Similarly, $F=\cap_{i} H_{i}$ iff $\mu_{F_{L}}(p)=i n f_{i \in I} \mu_{H_{L_{i}}}(p)$ for all $p \in Z_{L}$ and $\mu_{F_{U}}(p)=i n f_{i \in I} \mu_{H_{U_{i}}}(p)$ for all $p \in Z_{U}$ for all $p \in Z_{U}$.

\footnotetext{
Revised Manuscript Received on December 15, 2019
}

D. Vidhya, Department of Mathematics, Vels Institute of Science, Technology and Advanced Studies, Pallavaram, Chennai, India. E-mail: vidhya.d85@gmail.com
A complement $G^{\prime}$ of $G$ is defined by $\left(G_{L}^{\prime}, G_{U}^{\prime}\right)$ of $\lambda_{G_{L}}(q)=$ $1-\lambda_{G_{U}}(q)$ for all $q \in Z_{L}$ and $\lambda_{G_{U}}(q)=1-\lambda_{G_{L}}(q)$ for all $q \in Z_{U}$.

\section{Defintion 2.2 [8]}

A fuzzy rough set $K=\left(K_{L}, K_{U}\right)$ in $Z$ is defined by $K_{L}: X_{L} \rightarrow I$ and $K_{U}: X_{U} \rightarrow I$ with $K_{L}(x) \leq K_{U}(x)$ for every $x \in X_{U}$. The group of all fuzzy rough sets in $\operatorname{Zis} F R S(Z)$.

Definition 2.3 [8]

Any fuzzy rough topology on a rough set $X$ is a family $T$ of fuzzy rough sets in $X$ which satisfies the following conditions:

$\begin{array}{ll}\text { 1) } & \tilde{0}, \tilde{1} \in T \\ \text { 2) } & \text { If } A, B \in T \text { then } A \cap B \in T \text { and } \\ \text { 3) } & \text { If } A_{j} \in T \text { for all } j \in J \text {, then } \\ & \cup_{j \in J} A_{j} \in T .\end{array}$

Therefore $X$ together with topology $T$ is called a fuzzy rough topological space. The members of $T$ are open and its complement is closed.

Defintion 2.4 [8]

$N=\left(N_{L}, N_{U}\right)$ is called neighbourhood of $A=$ $\left(A_{L}, A_{U}\right)$ iff there exists fuzzy rough open set $B=$ $\left(B_{L}, B_{U}\right)$ such that $A \subseteq B \subseteq N$.

\section{FUZZY ROUGH OPEN COVERS SEQUENCE AND COVERING SEQUENCE PRINCIPLES}

This section introduces the types of rough open cover sequences and covering principles and discuss their characterizations and relationship between them.

\section{Definition 3.1}

A collection $\Sigma=\left\{A_{i}: i \in J\right\}$ of rough sets in $X$ is fuzzy rough cover of $\tilde{1}$ if $\cup_{i \in J} A_{i}=\tilde{1}$. Each members of $\Sigma$ are open iff a collection $\Sigma$ is fuzzy rough open cover.

\section{Definition 3.2}

Each open cover has a finite subcover then the space is compact.

\section{Defintion 3.3}

If $\quad A_{n}=\left\{\left(x, A_{L_{m}}, A_{U_{m}}\right): n, m \in \mathbb{N}\right\} \quad$ for $\quad x \in$ $X A_{L_{m}}, A_{U_{m}} \in[0,1]$ and $n=m$ then $\left\{A_{n}\right\}_{n \in \mathbb{N}}$ is a fuzzy rough sequence.

\section{A. Fuzzy Rough Open Covers}

\section{Definition 3.1.1}

A covering sequence of a rough set $D=\left(D_{L}, D_{U}\right)$ is a collection $\Theta=\left\{\left\{A_{n}\right\}_{n \in \mathbb{N}}\right\}_{k \in J}$ if $\cup_{k \in J}\left\{A_{n}\right\}_{n \in \mathbb{N}}=D$. 
If each member of $\left\{A_{n}\right\}_{n \in \mathbb{N}}$ is open then $\Theta$ is said to be open cover sequence.

\section{Note 3.1.1}

Ddenotes the collection of all fuzzy rough open cover sequence of a space $X$.

\section{Definition 3.1.2}

If $\mathfrak{D}$ have finite element then it is said to be a fuzzy rough open subcover sequence. It is simply denoted $\mathfrak{D}_{X}^{f}$.

\section{Defintion 3.1.3}

If for each fuzzy rough open cover sequence $\mathfrak{D}^{f}$ has subcover sequence then the space is fuzzy rough compact sequence.

\section{Remark 3.1.1}

Product of finitely many fuzzy rough compact sequence space is fuzzy rough compact sequence space.

\section{Definition 3.1.4}

$\mathfrak{D}$ is said to be a locally finite open cover sequence if each fuzzy rough point has a neighbourhood that intersect only finitely many of the rough sets in fuzzy rough open cover sequence. It represents $D^{l f}$.

\section{Defintion 3.1.5}

If each fuzzy rough point lies in only finitely many member in $\mathfrak{D}$ then $\mathfrak{D}$ is said to be a finite fuzzy rough point open cover sequence. This collection simply denoted by $\mathfrak{D}^{p f}$.

\section{Remark 3.1.2}

From Note 3.1.1, Definition 3.1.2, 3.1.4 and 3.1.5, $\mathfrak{D}^{f} \subseteq \mathfrak{D}^{l f} \subseteq \mathfrak{D}^{p f} \subseteq \mathfrak{D}$.

\section{B. Fuzzy Rough Covering Sequence Principles}

$\mathfrak{U}$ and $\mathfrak{B}$ be families of fuzzy rough cover sequence.

\section{Definition 3.2.1}

If for each $\left\{C_{n}\right\}_{n \in \mathbb{N}}$ of member of $\mathfrak{A}$ there is a $\left\{E_{n}\right\}_{n \in \mathbb{N}}$ such that for each $n, E_{n} \in C_{n}$ and $\left\{E_{n}\right\}_{n \in \mathbb{N}}$ is a member of $\mathfrak{B}$ then the cover sequences is covering sequence principle. It is denoted by $\boldsymbol{C} \boldsymbol{S}_{p}(\mathfrak{A}, \mathfrak{B})$.

\section{Definition 3.2.2}

If each member of $\mathfrak{B}$ is contained in some member of $\mathfrak{A}$ then we say that $\mathfrak{B}$ is fuzzy rough refinement of $\mathfrak{A}$.

\section{Definition 3.2.3}

For every $\left\{M_{n}\right\}_{n \in \mathbb{N}}$ of member of $\mathfrak{A}$ there exists finite $\left\{N_{n}\right\}_{n \in \mathbb{N}}$ such that for each $n, N_{n} \subseteq M_{n}$ and $\cup\left\{N_{n}\right\}_{n \in \mathbb{N}}$ in $\mathfrak{B}$. We say that $\mathfrak{A}$ and $\mathfrak{B i s}$ finite covering principle $\boldsymbol{C S}_{\text {pfin }}(\mathfrak{A}, \mathfrak{B})$.

\section{Definition 3.2.4}

Finite union covering principle $\left(\boldsymbol{C} \boldsymbol{S} \boldsymbol{U}_{\text {pfin }}(\mathfrak{A}, \mathfrak{B})\right)$ is each $\left\{P_{n}\right\}_{n \in \mathbb{N}}$ of member of $\mathfrak{U}$ there is a $\left\{Q_{n}\right\}_{n \in \mathbb{N}}$ of finite sets such that for each $n, Q_{n} \subseteq P_{n}$ and $\left\{\cup Q_{n}\right\} \in \mathfrak{B}$.

\section{Remark 3.2.1}

$\mathfrak{A}, \mathfrak{B}, \mathfrak{E}$ and $\mathfrak{D b}$ fuzzy rough cover sequences then the following conditions are valid.
1) If $\boldsymbol{C} \boldsymbol{S}_{p}(\mathfrak{U}, \mathfrak{B}) \subseteq \boldsymbol{C} \boldsymbol{S}_{p f \text { in }}(\mathfrak{A}, \mathfrak{B})$ then $\boldsymbol{C} \boldsymbol{S}_{p}(\mathfrak{U}, \mathfrak{B}) \Longrightarrow$

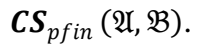

2) If $\boldsymbol{C} \boldsymbol{S}_{\text {pfin }}(\mathfrak{A}, \mathfrak{B}) \subseteq \boldsymbol{C S} \boldsymbol{U}_{\text {pfin }}(\mathfrak{A}, \mathfrak{B})$ then $\boldsymbol{C S}_{\text {pfin }}(\mathfrak{U}, \mathfrak{B}) \Longrightarrow \boldsymbol{C S U}_{\text {pfin }}(\mathfrak{U}, \mathfrak{B})$.

3) If $\boldsymbol{C} \boldsymbol{S}_{p}(\mathfrak{U}, \mathfrak{B}) \subseteq \boldsymbol{C} \boldsymbol{S}_{p \text { fin }}(\mathfrak{U}, \mathfrak{B})$ then $\boldsymbol{C} \boldsymbol{S}_{p}(\mathfrak{U}, \mathfrak{B}) \Longrightarrow$ $\boldsymbol{C S} \boldsymbol{U}_{\text {pfin }}(\mathfrak{U}, \mathfrak{B})$.

Proof: The Proof is direct from Definition 3.2.1,3.2.3 and 3.2.4.

\section{Definition 3.2.5}

Let $\Pi$ be one of covering principles and $\mathfrak{E}, \mathfrak{F}, \mathfrak{G}$ and $\mathfrak{H}$ be cover sequences. Define

$$
\begin{aligned}
& \text { 1) if(E } \subseteq(\mathfrak{H} \text { then } \Pi(\mathfrak{F}, \mathfrak{F}) \Rightarrow \Pi(\mathfrak{G}, \mathfrak{F}) \text {. } \\
& \text { 2) if } \mathfrak{F} \subseteq \mathfrak{H} \text { then } \Pi(\mathfrak{E}, \mathfrak{F}) \Longrightarrow \Pi(\mathfrak{G}, \mathfrak{H}) \text {. }
\end{aligned}
$$

We say that $\Pi$ is antimonotonic in the (1)and monotonic in the (2). These relations are indicated by the following diagram 1.1, where an arrow denotes an implication in the direction of the arrow:

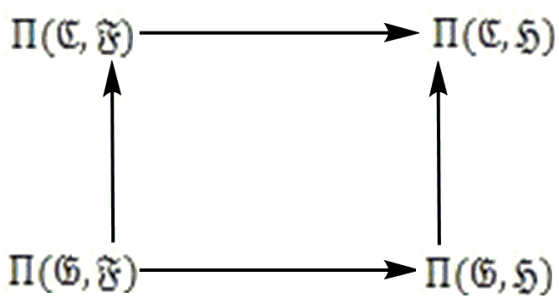

Figure 1

Note 3.1

For example considerП be $\mathbf{C S}_{p}(\mathfrak{C}, \mathfrak{F})$ then Figure 2 is illustrated :

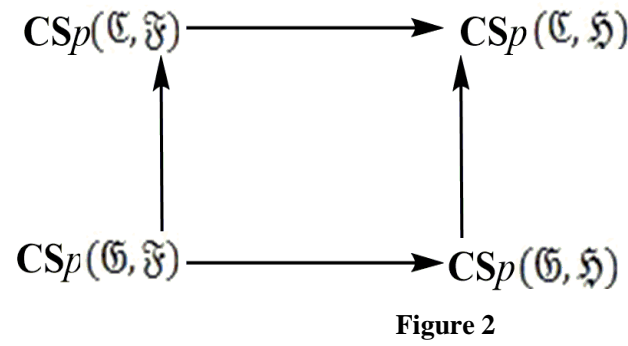

Similarly the rest of principles $\mathbf{C S}_{p f i n}$ and $\mathbf{C S U}_{p f i n}$ :

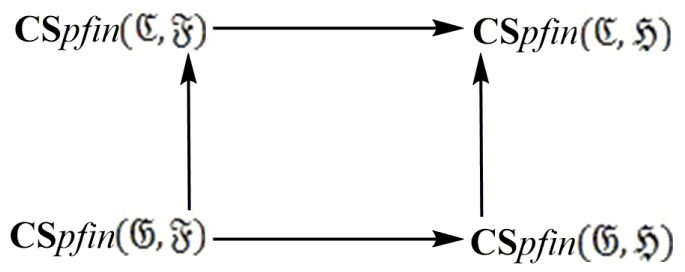

Figure 3 


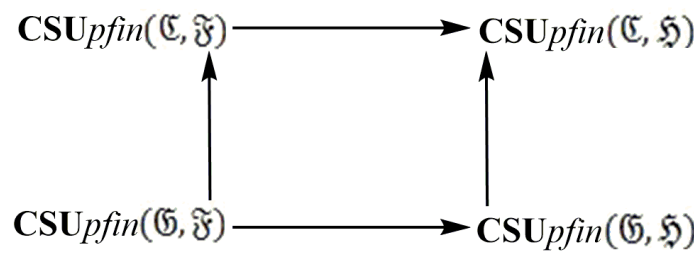

Figure 4

The summary of this section can be illustrated in figure 5 below.

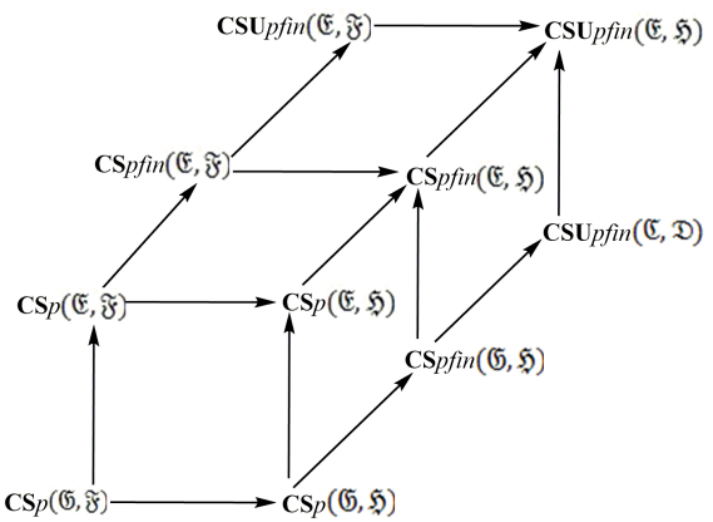

Figure 5

\section{RELATIONSHIP BETWEEN TYPES OF FUZZY ROUGH OOPEN COVER SEQUENCES AND COVERING SEQUENCE PRINCIPLES}

Tand $\mathfrak{B}$ represents one of the open covers of $\mathfrak{D}^{f}$ or $\mathfrak{D}^{\text {lf }}$ or $\mathfrak{D}^{p f} \quad$ or $\quad \mathfrak{D}$. Consider $\quad \boldsymbol{C S}_{p}(\mathfrak{U}, \mathfrak{B}) \subseteq \boldsymbol{C S}_{p f i n}(\mathfrak{A}, \mathfrak{B})$ and $\boldsymbol{C S}_{\text {pfin }}(\mathfrak{U}, \mathfrak{B}) \subseteq \boldsymbol{C S} \boldsymbol{U}_{\text {ffin }}(\mathfrak{U}, \mathfrak{B})$. Figure 6 is the combinations of open covers and covering principles

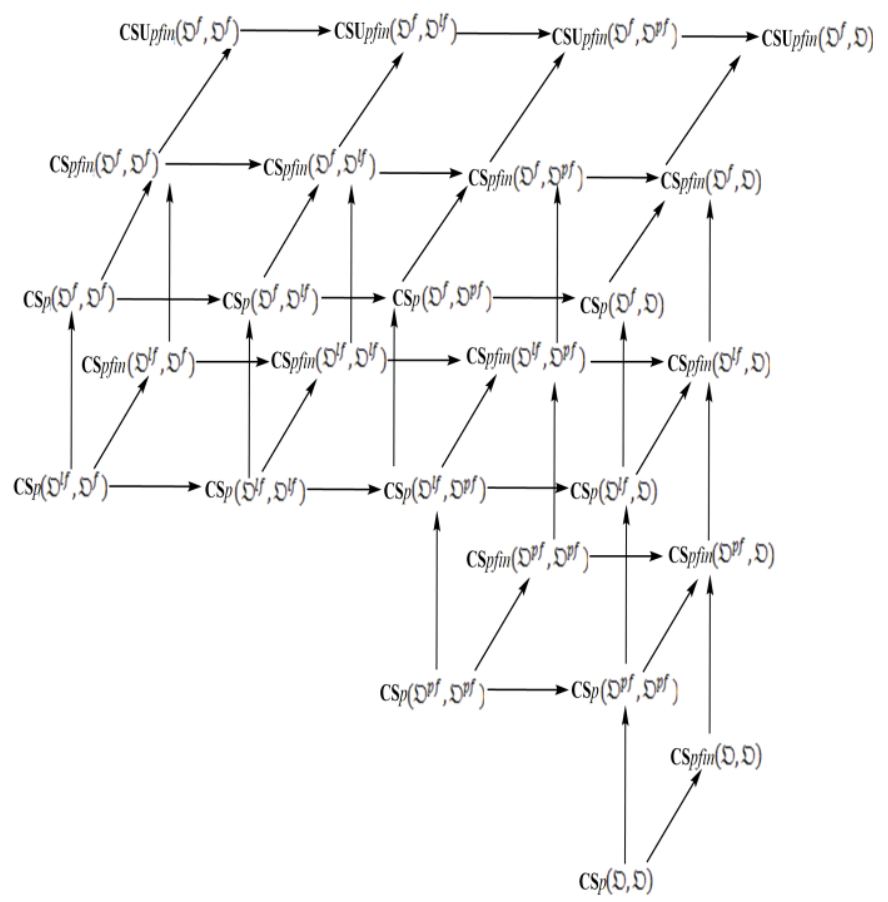

Figure 6

\section{CONCLUSION}

The figure 6 is also extended in soft fuzzy set, intuitionistic fuzzy set, fuzzy soft set etc. The concepts which are discussed in this paper have wide applications in network, printing technology and medical image processing.

\section{REFERENCES}

[1] ChangCL, "Fuzzy Topological Space", J. Math. Anal. Appl., 24,182190, 1968

[2] Mondal TK and Samanta SK, "Intuitionistic Fuzzy Rough Sets and Rough

Intuitionistic Fuzzy Sets", The Journal of Fuzzy Mathematics, 9 561582, 2001

[3] MathewBP and John SJ, "On Rough Topological Spaces", International Journal of Mathematical Archive, 3(9), 3413-3421, 2012

[4] NandaS and Majumdar S, "Fuzzy Rough Sets",Fuzzy Sets and Systems,

45, 157-160, 1992

[5] Pawlak Z, "Rough sets", Internat. J. Inform. Comput. Sci, 11(5), 341356 ,

1982.

[6] Smets P, "The Degree of Belief in a Fuzzy Event", Information Sciences,

25, 1-19, 1981.

[7] Sugeno M, "An Introductory Survey of Fuzzy Control", Information Sciences, 36, 59-83, 1985.

[8] VidhyaD, Roja E and Uma MK, “Algebraic Fuzzy Roguh Sheaf Group

Formed by Pointed Fuzzy Rough Topological Group", Int. J. Math. and Comp. Appl. Research, 4(1), 51-58, 2014.

[9] ZadehLA, "Fuzzy Sets", Information and Control, 8, 338-353, 1965. 